\title{
Automatic mediation in group problem solving
}

\author{
DAVID STODOLSKY \\ University of Louisville, Louisville, Kentucky 40292
}

\begin{abstract}
Conflicts over allocation of speaking time often occur in both formal and informal conferencing. In a problem solving experiment, an automatic mediator resolved conflicting requests during telephone conferencing. It combined inputs signaling intention to speak, using preprogrammed rules that then selected the new speaker. Teams of four undergraduate students repeatedly called upon the mechanism to resolve conflicting requests. Participant and mechanism behavior were recorded for later evaluation by a protocol analysis program that checked data strings for correct syntax, simulated behavior of alternative resolution rules, and aggregated group and individual data for processing with a statistical package. This approach to group experimentation yields significant methodological advantages in terms of verification of correct experiment administration, reduction in experimenter effects, and promotion of self-direction by participants.
\end{abstract}

This report presents a computer-based method of studying automatically mediated dialogue in the context of group problem solving. Automatic mediation refers to a procedure for group facilitation in which participants manage their own turn-taking behavior by signaling a computer mediator when they wish to speak. The mediator then stores these requests according to preprogrammed rules, subsequently displaying the name of the new speaker and activating that person's microphone or telephone. The experimental system reported here was developed for the purpose of exploring the effects of different rules for resolving conflicting requests to speak on the communication pattern and on the problem solving performance of groups. Specifically, it details the programs developed at the University of California, Irvine, School of Social Science, for administering a group problem solving experiment and processing the resultant data. The experimental system, like the procedure for resolving conflicting requests to speak, permitted participants to control the sequence of experimental events. A discussion of how the experiment is administered and how the resulting data are checked for accuracy and aggregated by a protocol analysis program is presented.

Recent reports by Brown (1979) and Cappella and Streibel (1979) have presented automated procedures for analyzing voice chronography data. Their reports emphasize the utility of such talk-silence data for the study of diadic and multiperson communication. With automatic mediation, additional information is available in an anonymous request channel. This information signals people's intention to communicate, while in other methods of studying group communication, intentions must be inferred.

This work was supported in part by National Research Service Award 1 1:32 MH05164-03 from the National Institute of Mental Hcalth.
A fundamental assumption of this research procedure is that the independent channel for requests can facilitate coordination in group problem solving. In a typical conversation, only one person may be speaking at any given moment. In automatically mediated dialogue, however, many people may be responding anonymously and independently to the speaker. This increased density of responses ( $2^{n}$ vs. 1 of $n$ ) permits a more detailed assessment of the group communication pattern and the characteristics of the individuals in the problem solving group. In a preliminary report, a variable available only in the request channel seemed to significantly discriminate among groups of individuals with different personality characteristics (Stodolsky, Note 1). This variable measured the waiting time between when a request was submitted and when the person was to speak. It was found to correlate with the individual's pretest score on the Personal Report of Communication Apprehension (McCroskey, 1970). This finding supports the utility of the current approach, since measurement of this variable is not possible in the typical problem solving experiment.

Explicit mediation becomes more crucial in large social aggregates. In face-to-face groups, rules of procedure are of ten used to insure fair treatment of participants and deliberate consideration of the question at hand. Computer mediation of such groups has been limited to devices for collecting and aggregating votes or indications of preference. The automatic selection of speakers has been limited primarily to telecommunication situations in which a visual channel for coordination of turn taking is lacking. Another area in which automatic selection has been investigated is video conferencing, in which a number of individuals must share a single visual channel. These systems typically have extremely simple rules that activate a microphone or a television camera according to the amplitude of a voice signal from a speaker.

With automatic mediation, an electronic system is 
interposed among the parties in order to resolve procedural conflicts. In the experimental system described here, this channel is used only for the transmission of request-to-speak signals. The computer resolves conflicting requests, thereby facilitating turn taking by speakers and permitting greater attention to be focused on the problem. Both rules for resolving conflicting requests used in this study guaranteed each person an equal opportunity to participate. Some equal opportunity mechanisms, however, are substantially more stable and resistant to manipulation than are others.

\section{RATIONALE}

One rule for speaker selection, the first-in/first-out rule, is used in both manually and automatically facilitated groups. In large discussion groups, a facilitator often maintains a list of pending speakers. Names are added to the list as people request to speak and are crossed off as the people take their turns. The need for research in this area is illustrated by the relative sophistication of technical as opposed to social aspects of work on mediated voice conferencing. Cohen and ISI Research Staff (Note 2) describe research that, while producing a number of technical advances in voice processing and encryption, uses a first-in/first-out rule for conferencing. This rule seems to work well when only a few people are waiting and when a time limit forces concise statements. A theoretical analysis, however, suggests that the first-in/ first-out rule can have severe disadvantages.

First, the crucial resource in group conferencing is the time available for discussion. A rule that focuses on turns is not likely to effectively utilize this resource. When a turn is not explicitly time limited, this inappropriate focus may result in a speech pattern with excessively long turns. A common result is that a person will have a number of points to comment on by the time his/ her turn arrives. Thus the interaction resembles a sequence of short speeches as opposed to a direct interchange of ideas. Even one person taking excessively long turns can force such a pattern, since others must respond similarly to maintain their fair share of speaking time. The discussion may become confused, since a response to a statement is likely to suffer substantial delay while other unrelated points are made. When turns are time limited, the situation may improve somewhat, but the first-in/ first-out rule is still not resistant to manipulation or panic. The rule does not specify a well-ordered joint strategy. That is, if each person seeks to maximize his/ her speaking time, the first-in/first-out rule collapses. In a situation in which persons request to speak immediately after their turn is completed, in an attempt to maximize their speaking time, the first-in/first-out rule collapses into a round robin rule with a static sequence (specified by the initial sequence of requests). Haley's (1964) results suggest that such a pattern is associated with pathology, at least in the types of family groups he studied. The equal time rule, by contrast, preserves a history of total speaking time, not merely the pattern of pending requests. This cumulative history permits a resolution of conflicting requests based upon a fair distribution of speaking time. Thus manipulation is precluded, and a joint strategy that attempts to maximize speaking time does not result in a collapse or panic.

\section{HYPOTHESES}

Three classes of hypotheses are considered when dealing with the effects of the rule chosen for automatic speaker selection. They concern the balance of participation, group emotional tone, and individual learning. The resolution rules directly affect the balance of vocal participation in the group, since the rules determine who will speak when requests conflict. The preliminary hypotheses are concerned with the magnitude of this differential in participatory balance. The first major hypothesis suggests a differential emotional impact upon individuals and the group as a unit, depending upon the rule used. The second major hypothesis suggests that improved participatory balance will affect individual learning and performance.

\section{Preliminary Hypothesis}

The preliminary hypothesis concerning participation is that the equal time resolution rule balances vocal participation more effectively than the first-in/first-out resolution rule. In first-in/first-out conferencing, the machine mediator places the group member's names on a list of people waiting to speak when they press their buttons. When the previous speaker finishes, the mediator selects the first person still on the list, that is, the one who has been waiting the longest to speak. In equal time conferencing, the mediator places the group member's names on a list of people waiting to speak when they press their buttons. When the previous speaker finishes, the mediator selects the person still on the list who has spoken the least thus far.

There are two methods of determining the balancing effects of the equal time speaker selection procedure. The first is experimental comparisons; balance in both elapsed speaking time and in number of speaking turns is compared across the experimental conditions. The balance of participation can also be compared with that typical of unmediated dialogue. Stodolsky (1979) found significant balancing as measured by the distribution of turns resulting from mediation by the equal time resolution rule as compared with unmediated dialogue.

The second method is simulated comparison: During data analysis of one speaker selection algorithm, the other is simulated. At each point a speaker was selected, the data analysis program determines whether the simulated algorithm would have resolved the conflicting floor 
requests differently. This differential resolution measure indicates to what degree the different algorithms choose the next speaker differently.

\section{Major Hypotheses}

The first major hypothesis concerning emotional tone is that the equal time resolution algorithm will counter communication apprehension by aiding persons unaccustomed to public speaking in gaining a turn to speak (for example, by putting them ahead of others in the speak ing order so they get a turn before they change their minds). The hypothesis is tested by comparing communication apprehension scores from a pretest with degree of participation as measured by the fraction of group conferencing time used and fraction of statements made by that group member.

Additional measures of emotional tone are derived from sociometric ratings of group members on a posttest. Each group member rated the individuals in the group, self included, on degree of leadership, quality of ideas, degree of participation, amount of self-confidence, and degree of influence exerted on the group. These ratings are related to pretest scores for differences between conditions. Objective measurements taken during the experiment, such as elapsed speaking time, are compared with group ratings for discrepancy between real and perceived effects, such as participatory balance. Posttest ratings also permit measurement of accuracy of selfperceptions vs. other perceptions on sociometric measures.

The emotional tone of the group as a unit is evaluated by examining a matrix of speaker transitions. Haley (1964) found that stereotyped turn-taking patterns were indicative of pathology in family groups. In his study, each of the first- through seventh-order transitions was highly significant in discriminating between pathological and normal families; pathology was assessed by independent tests and interviews. This type of analysis could characterize a group engaged in fruitless argument. In such a group, we might find the speaker transition matrix indicating that Person $A$ was always followed by Person B, who in turn would be followed by Person A. Such a continuing back-and-forth exchange without any comments by other group members could indicate poor emotional tone.

The second major hypothesis is that balanced participation increases individual learning and performance. Individual learning and performance were measured by effective utilization of available information presented during problem solving. Participants learn an optimal strategy for solving a permutation and combination problem that is demonstrated by a robot player. The strategy is based upon clues indicating the consistency of prior guesses with the correct answer.

The demonstrated optimal strategy is one that uses all information in previous clues. The individual guesses are objectively scored with reference to this same criterion by a protocol analysis program. The program scores both inconsistency in choice of object and incon- sistency in ordering the objects chosen. A minimum of inconsistency indicates effective strategy learning. The performance of the group as a unit is defined as the sum of individual performances. The group's task is to teach its members the solution strategy.

\section{THE CONFERENCE CALL EXPERIMENT}

\section{Method}

Participants and Design. Participants in the experiment described here were undergraduates at the University of California, Irvine, in the fall of 1975. An attempt was made to match groups with respect to both sex and communication apprehension (McCroskey, 1970). The multivariate design included three games, with short rest periods interspersed.

The task was a logic problem in which the goal was to correctly select and order four of six objects. This task was patterned after a popular game called Mastermind. ${ }^{2}$ The game proceeds by alternation of clues presented by the computer and participant guesses. Each of the guesses consisted of four choices from a set of colors. Four different colors had to be chosen or an input error was indicated by a message and the participant had to choose again. The clues were a preset sequence of guesses with associated feedback. The preset sequence was the independent guess of a "robot." Thus each group was exposed to an identical set of clues, even if their own guesses were markedly different. Each game consisted of four guesses, with feedback indicating the number of correct objects selected and the number of correct objects selected in the correct location. After individuals submitted their guesses, they had an opportunity to discuss their choices. Then the robot's guess and the feedback to it were presented. Finally, they were permitted to study these clues until they were ready to submit their next guess.

Apparatus. The conference call experiment (Stodolsky, 1976) was run on a PDP- $11 / 20^{2}$ computer running DOS $8-08$. A Digital Equipment Corporation terminal (VT-05) was used as a control console. The console information was available in individual rooms that contained a video display and a telephone handset. The telephone handset included a push-to-talk button that signaled that the person wished to speak or go on to the next part of the experiment. Wires from the telephone handset and the pushbutton were connected to cables that ran through the wall to the adjoining computer room. In each booth, a page of instruction reminders summarizing the functions of the procedures and apparatus was available. The dialogue was recorded on both a videotape machine, which also recorded the displayed video information, and on a dual-track tape recorder. Pretests were processed on a Digital Equipment Corporation PDP-11/40 under the response time sharing system (RSTS). ${ }^{2}$

Procedure. Participants were administered the pretest about 2 weeks prior to the experimental session. The pretests were automatically scored and then ordered from least to most apprehensive by a BASIC PLUS program. Matched groups were then formed by selecting one participant from each quartile (for groups of four). When participants arrived for the experiment, their names were entered at the control console and each was directed to a different room. The first page of instructions became available on the display when all participants had arrived and indicated their readiness to proceed by pressing their buttons. Successive pages of instructions were presented in the same manner. During the preliminary phase of the experiment, all microphones were open; thus participants could speak to each other and discuss the instructions as they read them. During telephone conferences, they helped each other to operate the apparatus and to solve the problem. Request-to-speak commands generated by pressing a switch activated the telephones according to a preset conflict resolution rule administered by the computer. The machine interpreted this rule and activated one participant's telephone while simultaneously presenting that person's name on a television display visible to all participants. 
Once the names of the participants were typed into the control terminal by the experimenter, only the system interacted with the participants until the experiment terminated; that is, the experimenter did not intervene during the experimental session. Lack of intervention reduced experimenter effects and freed the experimenter for other activities. The computer presented instructions, mediated a get-acquainted conversation, assigned names to booths, presented the problem, mediated discussion, called a special discussion period if procedural errors were made repeatedly, accepted responses, recorded detailed data on its own behavior as well as on that of the participants, and finally, terminated the experiment by asking participants to report to a room for debriefing.

\section{Results}

Data analysis. In this experiment, the operational program was written in FORTRAN, with data stored in arrays and manipulated according to mathematically oriented statements. The protocol analysis program was written in SNOBOL, using strings (i.e., sequences of characters) for data storage. These strings of information were manipulated by pattern matching and concatenation. Certain crucial variables, such as the state of the list of waiting speakers, are printed by the speaker selection routines for both error checking and detailed data analysis. This mass of data, over 100,000 characters/ session, contributes to the difficulty of data analysis. The alternative approach, the use of a single program to run the experiment and aggregate the data for future analysis, would likely be less time-consuming. The rigorous control of errors, however, would be harder to achieve.

Data tape. The data tape is the repository of all information on the behavior of the operational program and the participants. Figure 1 indicated the degree of detail available. Since various subroutines of the operational program transmit data to the tape, there is considerable redundancy and a certain degree of asynchrony. About $27 \mathrm{sec}$ of events are detailed in Figure 1.

The first line of Figure 1 indicated that the message "Waiting for new speaker" appeared on the display $3,488.073 \mathrm{sec}$ after the clock was started. The elapsed time is given as the last parameter in each line of the example. Within $.1 \mathrm{sec}$, the switch subroutine indicated that the switch in Booth 1 (Kathryn) was depressed (switch state equals 1). About .1 sec later, first-in/ first-out reports stacking Name 1 (Kathryn) at Stack Index 2. Since the stack is always "popped" (all stacked names are moved one closer to zero), the first request enters the second stack location before the new speaker is selected. The next output from the microphone control subroutine indicates the microphone element in Booth 1 (Kathryn) was activated. This was momentarily followed by the name display, "New speaker Kathryn F.," indicating the start of a turn.

Protocol analysis. The function of the protocol analysis program is to produce a chronological description of the experimental session and aggregate data arrays characterizing the individual behavior of each participant and the behavior of the group as a whole. The program

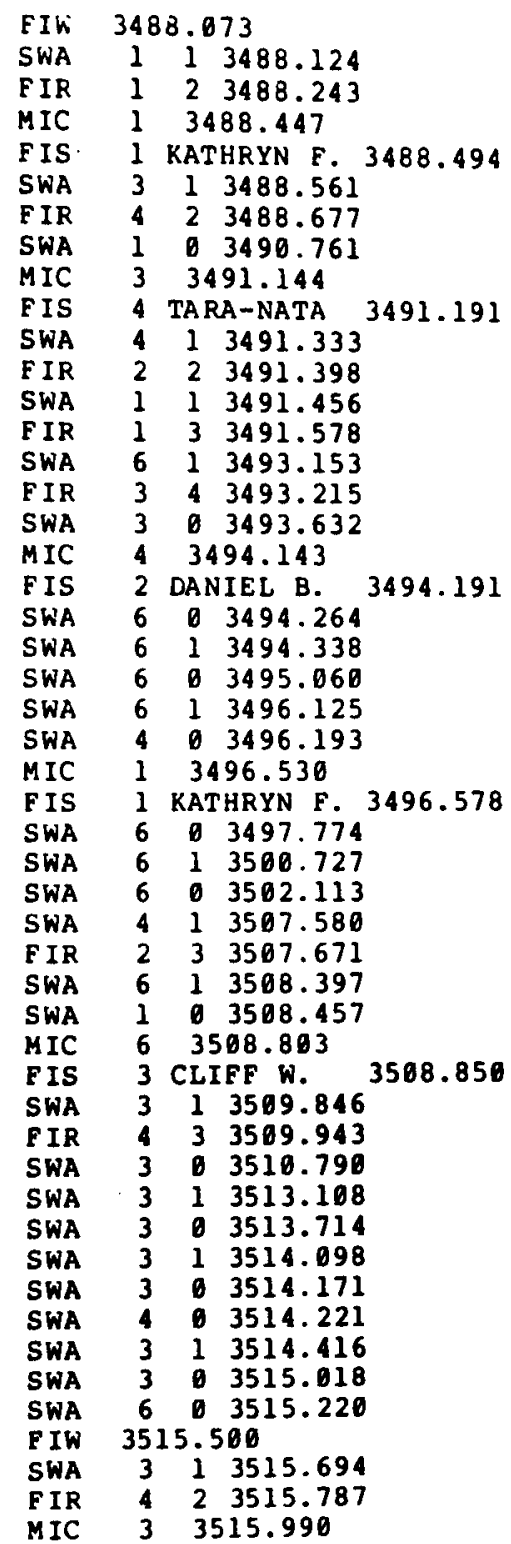

Figure 1. Data tape example.

also simulated the behavior of the operational program in order to compute differential resolutions of floor requests and to uncover errors in administration. The protocol analysis program for analyzing the first-in/ first-out data simulates the equal time condition. The protocol analysis program for analyzing equal time data simulates the first-in/first-out condition to compute differential resolutions and simulates the equal time rule to check for timekeeping errors. Both programs are written in the SITBOL (Version 4A) dialect (Gimpel, 1972) of SNOBOL (Griswold, Poage, \& Polonsky, 1971).

The protocol analysis program for the first-in/first-out data is primarily composed of a series of subroutines that are activated during a pattern-matching process. The pattern-matching process as a whole is useful in that it 
can only succeed when a sequence of data output conforms, to the last character, to the specified experiment syntax. Before attempting a match, the program reads in all data lines that fall between two successful guesses. It concatenates these elements (see Figure 1) and applies the pattern "CONP FEEDBACK STUDY CHOICES RPOS (0)" if the elements do not precede the first guess of a game. This pattern will match conferencing data, followed by feedback data, followed by data from a study period, and terminated by guessing data. The pattern will match only if the subpatterns match in such a way as to absorb the entire string of data, indicated by the primitive pattern RPOS(0) (right position zero). Each of these pattern elements may be composed of other detailed patterns. For instance, the pattern CHOICES is composed of outputs from the operational subprogram IGSS (individual guess collection) followed by an arbitrary number of alternate conferencing patterns and IGSS outputs. This accommodates any sequence involving error conferences. In the case of CHOICES, the fourth-layer subpattern specifies valid output from IGSS.

Group data. The next part of the program outputs group statistics for the period preceding each guess and for the introductory conference (Table 1). During the analysis, the mediator is treated as another participant, Participant 0 . Many of the measures of mediator behavior correspond to individual behavior. For instance, the number of waits indicates how many times the message "Waiting for new speaker" appeared (Table 1). For an individual, the corresponding measure, "selected," indicates how many times that person gained the floor; in the case of the mediator, it indicates nonoverlapping floor requests. The next column gives the number of conferences in each preguess period. The speaking time indicates how long all group members spent speaking.
Differential resolutions indicate the number of times the alternate speaker selection algorithm would have chosen a speaker different from the speaker chosen by the current selection algorithm. This determination occurs only at the .1-sec moment of speaker selection. If two speakers requested the floor from the mediator and their requests were separated by more than $.1 \mathrm{sec}$, no conflict would exist. By the time the second request was entered, the floor would have been granted to the first requestor. Conflict resolution occurs only when at least two requests are pending. During the total of about $25 \mathrm{~min}$ of mediated conferencing, there were $39 \mathrm{con}$. flicts. In 2 of these, three people had floor requests pending, and in the remaining 37 , two persons contested the floor. Summing the differential resolutions from Table 1 indicates that 18 conflicts would have been resolved differently by the alternate algorithm for this group of four. This assumes no effect of the rule definition in the instructions and of differential resolutions upon speech request behavior. Thus alternative conflict resolution rules can strongly influence speaking order in groups as small as four persons. According to the protocol analysis simulation, half of these would have been resolved differently had the mediator utilized the equal time rule as opposed to the first-in/first-out rule. This finding suggests that automatic conflict resolution can significantly alter the pattern of turn taking in smallgroup discussion. The effect can be expected to be more profound as group size increases.

Time limits exceeded refers to the conference $(200 \mathrm{sec})$ or game $(600 \mathrm{sec})$ limits, not the turn-time limit. The waiting time indicates total amount of time the mediator held the floor and the message "Waiting for new speaker" appeared on the screen. Finally, total waiting time gives the sum for the entire experimental session of the waiting time given.

Table 1

Group Data

\begin{tabular}{|c|c|c|c|c|c|c|c|}
\hline $\begin{array}{c}\text { Conferencing } \\
\text { Period }\end{array}$ & $\begin{array}{c}\text { Number } \\
\text { of } \\
\text { Waits }\end{array}$ & $\begin{array}{l}\text { Number } \\
\text { of Con- } \\
\text { ferences }\end{array}$ & $\begin{array}{c}\text { Speaking } \\
\text { Time }\end{array}$ & $\begin{array}{c}\text { Differen- } \\
\text { tial Reso- } \\
\text { lutions }\end{array}$ & $\begin{array}{c}\text { Time } \\
\text { Limits } \\
\text { Exceeded }\end{array}$ & $\begin{array}{l}\text { Waiting } \\
\text { Time }\end{array}$ & $\begin{array}{c}\text { Total } \\
\text { Waiting } \\
\text { Time }\end{array}$ \\
\hline Introductory & 15 & 1 & 75.8 & 2 & 1 & 23.0 & 186.9 \\
\hline \multicolumn{8}{|l|}{ Game 1} \\
\hline Guess 1 & 12 & 1 & 82.1 & 3 & 1 & 18.8 & \\
\hline Guess 2 & 16 & 1 & 121.8 & 2 & 0 & 11.3 & \\
\hline Guess 3 & 23 & 1 & 183.6 & 3 & 1 & 14.5 & \\
\hline Guess 4 & 24 & 1 & 180.7 & 0 & 1 & 17.8 & \\
\hline \multicolumn{8}{|l|}{ Game 2} \\
\hline Guess 1 & 3 & 1 & 16.3 & 0 & 1 & 1.9 & \\
\hline Guess 2 & 27 & 1 & 174.7 & 1 & 1 & 22.4 & \\
\hline Guess 3 & 22 & 1 & 179.3 & 3 & $i$ & 19.1 & \\
\hline Guess 4 & 31 & 1 & 153.2 & 3 & 0 & 27.2 & \\
\hline \multicolumn{8}{|l|}{ Game 3} \\
\hline Guess 1 & 0 & 1 & .0 & 0 & 1 & .2 & \\
\hline Guess 2 & 25 & 1 & 140.5 & 0 & 0 & 20.5 & \\
\hline Guess 3 & 8 & 1 & 76.6 & 0 & 0 & 4.5 & \\
\hline Guess 4 & 8 & 1 & 80.8 & 1 & 0 & 5.1 & \\
\hline
\end{tabular}

Note-Times in seconds. 
Table 2

First-Order Speaker-to-Speaker Transitions

\begin{tabular}{lcrrrr} 
& \multicolumn{5}{c}{ To } \\
\cline { 2 - 6 } \multicolumn{1}{c}{ From } & 0 & 1 & 2 & 3 & 4 \\
& Mediator Kathryn & Daniel & Cliff & Tara-Nata \\
\hline 0 Mediator & 12 & 55 & 13 & 51 & 90 \\
1 Kathryn & 57 & 0 & 10 & 13 & 13 \\
2 Daniel & 19 & 16 & 0 & 4 & 8 \\
3 Cliff & 47 & 9 & 10 & 0 & 15 \\
4 Tara-Nata & 79 & 17 & 14 & 15 & 0 \\
\hline
\end{tabular}

The first-order speaker-to-speaker transitions are also output by this part of the program (Table 2). The number of times a speaker initiated conversational interaction is given by the top row. For instance, Tara-Nata followed the mediator (Number 0) 90 times during the session. This same speaker terminated a conversation, that is, was followed by the mediator, 79 times.

All terminations are given in the first column. The diagonal of the table indicates the number of times a speaker took two or more turns in a row. None did so in this group. The mediator did follow itself 12 times. This can only occur as an interconference event. Thus 12 times in the session, the mediator terminated a conference and began the next one. The number of times any speaker followed any other can also be read from the table. For instance, Kathryn followed Cliff nine times during the experimental session.

According to Table 2, a vast majority of the transitions occurring in the session were either to or from the mediator. Since the transition to the mediator can occur only if there are not requests pending, no conflicts can occur during such transitions. Also, because of the .1-sec cycle time used by the mediator, it is unlikely that there would be any conflict upon transitions from the mediator. In this sense, both speaker selection algorithms would be considered first-in/first-out selection algorithms; either one will give the floor to the first person who requests it. Thus the speaker selection algorithms are different only secondarily. The effective difference between them could be increased by permitting floor requests to cumulate over a period longer than $.1 \mathrm{sec}$ while waiting. In some groups, participants reported using the display "Waiting for new speaker" to indicate that the previous speaker had terminated. This behavior would account for the large numbers in the first row and column of the transition matrix and for the small number of total conflicts.

The data on conversational initiation were analyzed to test the hypothesis that unresolved transitions in mediated groups have the same pattern as those observed in free interaction. This result is important because of the rather limited progress in instrumentation for social-psychological experimentation. A major question posed by the availability of automatic facilitation apparatus is its effectiveness vs. manual procedures in group facilitation. This question could be investigated by comparing automatic and manually facilitated groups on outcome measures; however, it would do little to elucidate the underlying process. More effective utilization of resources would result from examining the impact of a minimal automatic mediator. First, the external validity question could be addressed by examining the obtrusiveness of the experimental environment. If interaction patterns with minimal automatic mediation do not deviate significantly from those of free interaction, then the minimal system can be used as a baseline for further investigation. A highly instrumented baseline would permit more economical investigation by eliminating the need for manual transcription of interaction data. Identifying this minimal system would also have theoretical implications by isolating mediation parameters unlikely to be responsible for therapeutic change. If a mediation procedure is highly transparent in use (i.e., if it does not alter interaction parameters), then it will be an effective analogue of free discussion. One interaction parameter that has been studied extensively is relative initiation rate.

In the analysis presented here, the assumption that unresolved transitions in automatically mediated conferences are equivalent to initiations in free discussion is examined. The initiation rates are compared with those predicted by Horvath's (1965) turn-taking model. Table 3 presents number of turns initiated from the waiting state (first row in transition matrix) during conferencing for four groups.

Horvath's (1965) model assumes each person has the same constant probability, $\mathrm{P}$, of initiating the next comment. It is further assumed, however, that the group rapidly reaches a hierarchical equilibrium, as if members wait for the persons preceding them in the hierarchy to speak.

Thus, the probability of a turn taken by the highest participator is represented as $P$; the second person speaks with a probability of $(1-P) P$, the third, $(1-P)^{2} P$, the nth, $(1-P)^{N-1} P$. If no one down the hierarchy speaks, then the highest participator gets another chance. The probability of the first-ranking person's speaking is the sum of probabilities from each chance, $P+P(1-P)^{N}+P(1-P)^{2 N}+\cdots$. The sum of the infinite series is $P /\left[1-(1-P)^{N}\right]$. This is equivalent to $A$, the fitted proportion of initiations by the highest participator in the Stephan and Mishler (1952) equation. The second-ranked person's probability becomes $A(1-P)$, the third's $A(1-P)^{2}$, and so on. If $(1-P)$ is replaced by $r, A=(1-r) /\left(1-r^{n}\right)$ and proportion of turns taken by $i=A r i-1$. Thus, a single parameter $P$, the probability of initiating the next comment, can be used to deduce the Stephan and Mishler equation. The fit to the data in Table 3 is good $\left[\chi^{2}(2)=5.46\right.$, n.s. $]$ (Stodolsky, 1979). The predicted initiation rates are all derived from the totals, where the mean $P$ is . 39 . 
Table 3

Fit of Stodolsky (1976) Data to Horvath (1965) Model: Unresolved Transitions (Interspersed Waiting Period)

\begin{tabular}{|c|c|c|c|c|c|c|c|c|c|c|}
\hline \multirow[b]{2}{*}{ Subject } & \multicolumn{2}{|c|}{$\begin{array}{c}\text { Group E7 } \\
\left(x^{2}=25.4\right)\end{array}$} & \multicolumn{2}{|c|}{$\begin{array}{c}\text { Group E8 } \\
\left(x^{2}=.1\right)\end{array}$} & \multicolumn{2}{|c|}{$\begin{array}{c}\text { Group E9 } \\
\left(x^{2}=5.83\right)\end{array}$} & \multicolumn{2}{|c|}{$\begin{array}{c}\text { Group E10 } \\
\left(x^{2}=.25\right)\end{array}$} & \multicolumn{2}{|c|}{$\begin{array}{l}\text { Total All Groups } \\
\left(\mathrm{x}^{2}=5.46\right) \\
\end{array}$} \\
\hline & Actual & Predicted & Actual & Predicted & Actual & Predicted & Actual & Predicted & Actual & Predicted \\
\hline 1 & 74 & 70 & 64 & 71 & 62 & 77 & 47 & 48 & 247 & 263 \\
\hline 2 & 50 & 43 & 44 & 43 & 53 & 47 & 31 & 29 & 178 & 162 \\
\hline 3 & 26 & 26 & 29 & 26 & 33 & 28 & 18 & 18 & 106 & 99 \\
\hline 4 & 5 & 16 & 20 & 16 & 21 & 17 & 10 & 11 & 56 & 61 \\
\hline Total & 155 & & 157 & & 169 & & 106 & & 587 & \\
\hline
\end{tabular}

Note-Subjects are ranked in order of participation. $P=.39$ for all data.

Table 4

Individual Data

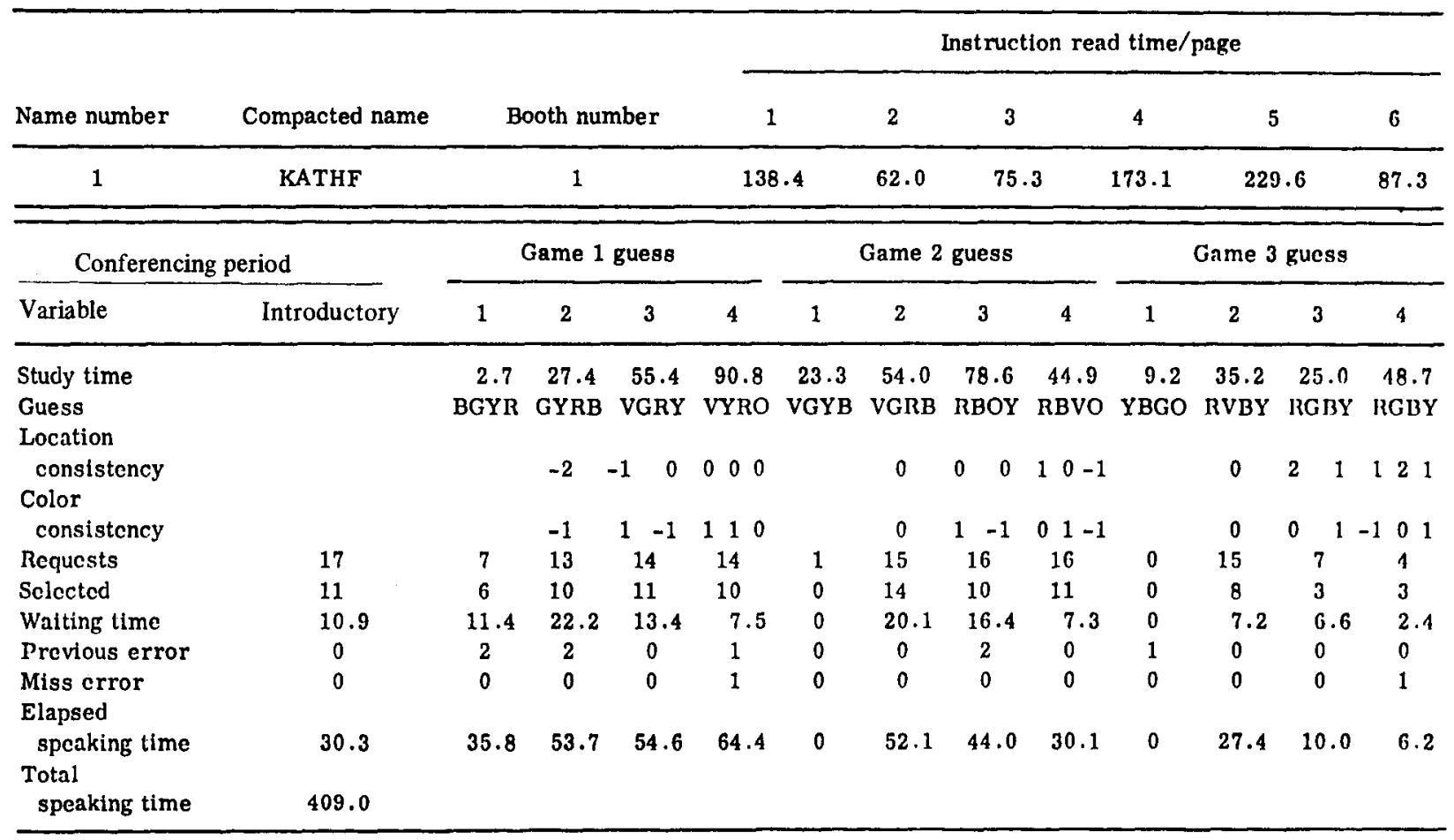

Note-Times in seconds.

Individual data. The last part of the program prints the data of each participant (Table 4). The first line gives the name order number, followed by the name compacted to five characters, the booth number, and the instruction reading times for each page of instructions. The name must be compacted in order to be read by the Statistical Package for the Social Sciences (Nie, Bent, Hull, 1970) available on the Digital Equipment Corporation PDP-10 computer (Matzek, Note 3).

The study time before each guess is given to the nearest $.1 \mathrm{sec}$ on the next line. The guess selected is given next, followed by the location and color consistency scores. There is one consistency score for each of the previous guesses in a game. A score of zero indicates complete use of information in the previous guess. The line titled "selected" is the number of times the floor was granted by the mediator. The waiting time is the total number of seconds the participant waited between a request and being selected to speak. If the participant requested to speak but changed his/her mind before the previous speaker terminated, the time is not added to the total. The next two lines score the two types of guessing error. A "previous error" is charged when the participant tries to use the same color repeatedly in a guess. The "miss error" is charged when no color is chosen at all. The last line gives the elapsed speaking 
time during each period, and the final entry totals these times.

Chronological output. Each line of output starts with the booth number of the previous speaker and is followed by a number giving speaking time to $.1 \mathrm{sec}$. The next number is the selected new speaker's booth followed by the waiting time. The remainder of the line presents the stack, giving the booth number and request time for those remaining after the new speaker has been selected. The chronological output concludes with a list of all turns taken by each participant and the mediator to the nearest $.1 \mathrm{sec}$. Finally, group location and color consistency scores are given. This is defined as the total number of zeros in the consistency array of the participants.

\section{DISCUSSION AND CONCLUSIONS}

The utilization of an automatic mediator makes possible independent measurement of intention to speak and actual speech. When the request channel and the speech channel are combined, as is typical, these measurements are confounded. The type of speech request and speech data presented here can facilitate the testing of social interaction theories.

Many formal procedures, both manual and automated, use a first-in/first-out rule as the primary mechanism for structuring interaction. Alternative automatic mediation rules can strongly influence the sequence of turn taking in teleconferencing. This effect was demonstrated in a four-person group and should be more pronounced in larger groups. Substantial improvements in participatory balance and the quality of interaction may be achieved by appropriate choice of conference mediation rules.

In an environment with visual isolation, such as that used in the conference call experiment, the information transmitted through the mediation mechanism can avoid the necessity for the rules' use in many cases. It is interesting that the participants in the experiment avoided using the conflict resolution algorithms by waiting for the previous person to release the channel, thereby causing a message "Waiting for new speaker" to be presented to the group before requesting to speak. Thus, potential conflict at each speaker-to-speaker transition was circumvented. This minimization of conflict is also manifested in the subjective reports of participants: When the mediation mechanism was operating correctly, it appeared transparent to participants, that is, it did not impede the flow of communication between group members. A vast majority of persons indicated that they had been fairly treated by the automatic conflict resolver.
When the mediation mechanism failed to respond correctly, however, a great deal of frustration was felt by certain persons, with an impact upon the group as a whole. These findings suggest that groups can rapidly adapt to the use of an automatic mediator; a fair mechanism becomes obstrusive only when it malfunctions.

\section{REFERENCE NOTES}

1. Stodolsky, D. Waiting-to-speak time, communication apprehension level, and information processing load in group problem solving. Manuscript submitted for publication, 1980.

2. Cohen, D., \& ISI Research Staff. Network secure communication. In ISI Research Staff (Eds.), A research program in computer technology, annual technical report, July 1975-June 1967 (Tech. Rep. ISI/SR-76-6). Marina del Rey, Calif: University of Southern California, Information Sciences Institute, July 1976.

3. Matzek, M. A. The University of Pittsburgh, SPSS-10 (statistical package for the social sciences) (Version 5.02.2). Pittsburgh: University of Pittsburgh Computer Center, December 5 , 1974.

\section{REFERENCES}

Brown, E. F. Apparatus for collecting voice chronography data. Behavior Research Methods \& Instrumentation, 1979, 11, 553557.

Cappella, J. N., \& Streibel, M. J. Computer analysis of talksilence sequences: The FIASSCO system. Behavior Research Methods \& Instrumentation, 1979, 11, 384-392.

Gimpel, J. F. SITBOL, Version 1.0. Holmdel, N.J: Bell Telephone Laboratories, Inc., 1972.

Griswold, R. E., Ponge, J. R., \& Polosky, I. P. The SNOBOL 4 programming language (2nd ed.). Englewood Cliffs, N.J: Prentice-Hall, 1971.

Haley, J. Research on family patterns: An instrument measurement. Family Process, 1964, 3, 41-65.

Horvath, W. J. A mathematical model of participation in small group discussions. Behavioral Science, 1965, 10, 164-166.

McCroskey, J. C. Special reports: Measure of communicationbound anxiety. Speech Monographs, 1970, 37, 269-277.

Nie, N., Bent, D. H., \& Hull, C. H. SPSS, statistical package for the social sciences. New York: McGraw-Hill, 1970.

Stephan, F. F., \& Mishler, E. G. The distribution of participation in small groups. American Sociological Review, 1952, $17,598-608$.

Stodolsky, D. Machine-mediated group problem-solving: Therapy, learning, performance (Doctoral dissertation, University of California, 1976). Dissertation Abstracts International, 1976, 37, 1949B. (University Microfilms No. 76-19,633)

Stodolsky, D. Group problem-solving with automatic mediation. Proceedings of the Annual Convention of the Association for Development of Computer-Based Instructional Systems, $1979,3,781-794$.

\section{NOTES}

1. Mastermind is a registered trademark of Invicta Plastic Limited.

2. PDP and RSTS are registered trademarks of the Digital Equipment Corporation. 